\title{
Experimental evidence for Coulomb charging effects in an open quantum dot at zero magnetic field
}

\author{
C.-T. Liang, M. Y. Simmons, C. G. Smith, G. H. Kim, D. A. Ritchie and M. Pepper \\ Cavendish Laboratory, Madingley Road, Cambridge CB3 OHE, United Kingdom
}

(March 16, 2018)

\begin{abstract}
We have measured the low-temperature transport properties of an open quantum dot formed in a clean one-dimensional channel. For the first time, at zero magnetic field, continuous and periodic oscillations superimposed upon ballistic conductance steps are observed when the conductance through the $\operatorname{dot} G$ exceeds $2 e^{2} / h$. We ascribe the observed conductance oscillations to evidence for Coulomb charging effects in an open dot. This is supported by the evolution of the oscillating features for $G>2 e^{2} / h$ as a function of both temperature and barrier transparency.
\end{abstract}

PACS numbers: 73.40.Gk, 73.20.Dx

The use of sub-micron surface gates fabricated over GaAs/AlGaAs heterostructures to electrostatically squeeze [1] the underlying two-dimensional electron gas (2DEG) into various shapes, has been proved a powerful tool to realize low-dimensional electron systems. By employing this technique, it is possible to define a quantum dot which confines electrons in an isolated region within a 2DEG. Consider a lateral quantum dot [2] weakly coupled to the source and drain contacts where the tunneling conductance through the $\operatorname{dot} G$ is low, $G \ll 2 e^{2} / h$. If the thermal smearing $k_{B} T$ and the chemical potentials in the leads are much smaller than the Coulomb charging energy $e^{2} / C$ which is required for adding an extra electron to the quantum dot, transport through the dot is inhibited. This is the Coulomb blockade (CB) of single electron tunneling [3]. It has been demonstrated [4] that transport through small quantum dots is determined by Coulomb charging effects [5] as well as zero-dimensional (0D) quantum confinement effects [6].

A versatile quantum dot may be defined by two pair of split-gates which introduce two quantum point contacts acting as the entrant and exit barriers to the dot, and two side-gates which are used to deplete electrons within the dot [7]. It has been reported that at zero magnetic field Coulomb charging effects only occur when the conductance of the two quantum point contacts, and of the quantum dot as a whole, falls below $2 e^{2} / h$ 胿 9 . No oscillations were found when either or both of the quantum point contacts were set to above $G>2 e^{2} / h$ so that the dot was open to the 2DEG reservoirs [7 9]. Thus at present, it is widely accepted that at zero magnetic field, the conductance $2 e^{2} / h$ is the upper limit for which Coulomb charging effects can occur [10]. Nevertheless, previously we presented evidence that at zero magnetic field, Coulomb charging effects can occur even when the total conductance through the dot is greater than $2 e^{2} / h$, provided that the fully transmitted one-dimensional (1D) channels only partially screens the Coulomb charging and there is little inter 1D subband scattering 11,12]. In this work the quantum dot was formed by an impurity potential, and precise control over the tunnel barriers was not possible, hindering detailed studies of this effect.

Motivated by these early results, we have designed a new type of open quantum dot device with tunable barriers. In this Letter, we report low-temperature conductance measurements of an open quantum dot device in which impurity scattering is negligible. Due to the unique design of our devices fabricated on an ultra highquality high mobility electron transistor (HEMT), we present clear evidence, for the first time, of Coulomb charging effects in an open quantum dot at zero magnetic field. This is supported by the temperature and barrier transparency dependence of the observed periodic conductance oscillations for $G>2 e^{2} / h$.

The two-layered Schottky gate pattern shown in the inset of Fig. 1 was defined by electron beam lithography on the surface of a high-mobility $\mathrm{GaAs} / \mathrm{Al}_{0.33} \mathrm{Ga}_{0.67} \mathrm{As}$ heterostructure T258, $157 \mathrm{~nm}$ above a 2DEG. There is a $30 \mathrm{~nm}$-thick layer of Polymethylmethacrylate (PMMA) which has been highly dosed by an electron beam, to act as a dielectric 13 between the split-gate (SG) and three gate fingers $(\overline{\mathrm{F}} 1, \mathrm{~F} 2$, and F3) so that all gates can be independently controlled. The carrier concentration of the 2DEG was $1.6 \times 10^{15} \mathrm{~m}^{-2}$ with a mobility of $250 \mathrm{~m}^{2} / \mathrm{Vs}$ after brief illumination by a red light emitting diode. The corresponding transport mean free path is $16.5 \mu \mathrm{m}$, much longer than the effective $1 \mathrm{D}$ channel length. Experiments were performed in a dilution refrigerator at $T=50 \mathrm{mK}$ and the two-terminal conductance $G=d I / d V$ was measured using an ac excitation voltage of $10 \mu \mathrm{V}$ at a frequency of $77 \mathrm{~Hz}$ with standard phase-sensitive techniques. In all cases, a zero-split-gatevoltage series resistance $(\approx 900 \Omega)$ is subtracted. Two samples, at five different cooldowns, show similar characteristics and measurements taken from one of these are presented in this paper.

Trace 1 in figure 1 shows the conductance measurements $G\left(V_{S G}\right)$ as a function of split-gate voltage $V_{S G}$ 
when all finger gate voltages $V_{F 1}, V_{F 2}$, and $V_{F 3}$ are zero. We observe conductance plateaus at multiples of $2 e^{2} / h$, with no resonant feature superimposed on top, as expected for a clean 1D channel. When the channel is defined at $V_{S G}=-1.132 \mathrm{~V}$, five quantized conductance steps are observed when each one of the finger gates is swept while the others are earthed to the 2DEG as shown in traces 2-4 (Fig. 1). These experimental results demonstrate that we have a clean 1D channel in which impurity scattering is negligible. Periodic resonant features, as shown later, are only observed when large negative voltages are applied to both F1 and F3.

We can define a lateral quantum dot by applying voltages on SG, F1, and F3 while keeping F2 earthed to the 2DEG. Trace 1 in Fig. 2 shows the gate characteristics $G\left(V_{S G}\right)$ for $V_{F 1}=-1.941 \mathrm{~V}$ and $V_{F 3}=-1.776 \mathrm{~V}$ at $T=50 \mathrm{mK}$. Striking periodic and continuous conductance oscillations superimposed on ballistic conductance steps are observed. We ascribe the observed conductance oscillations for $G<2 e^{2} / h$ to Coulomb charging effects [7]. The observed periodic conductance oscillations for $G>2 e^{2} / h$ are unexpected and are the main subject of this paper. Unlike lateral quantum dots whose tunnel barriers are defined by two pair of split-gates, in our system, the tunnel barriers arise from depletion from overlying finger gates. This causes a large barrier thickness so that we do not observe well-isolated single electron tunnelling beyond pinch-off in our case. In contrast to the well-quantized conductance plateaus shown in Fig. 1 (a), applying voltages to $\mathrm{F} 1$ and $\mathrm{F} 3$ results in conductance steps that are not as flat or well quantized. With the finger gates earthed to the 2DEG, the channel pinches-off at $V_{S G}=-1.8 \mathrm{~V}$ compared with $V_{S G}=-0.7 \mathrm{~V}$ when $V_{F 1}=-1.941 \mathrm{~V}$ and $V_{F 3}=-1.776 \mathrm{~V}$. Thus as voltages are applied to F1 and F3, the lateral confinement weakens and the conductance steps become less pronounced. The conductance steps also deviate from their quantized values. The most likely reason for this effect is due to the introduction of two tunnel barriers which enhances backscattering in the channel, thereby reducing the transmission probability of $1 \mathrm{D}$ channels [14] to be less than 1 .

Previously in a lateral quantum dot [7] 9] has been observed that Coulomb oscillations increase in height and decrease in width as the conductance decreases. This increase in height arises from an accumulation of the electron wave function in the dot, giving rise to resonant coherent effects, as the dot becomes isolated from the source and drain contacts. From figure 2 we can see that no such increase in height is observed in our system as the conductance is decreased. We believe that the thicker tunnel barriers in our system make it more difficult for the electrons to tunnel out such that the electron lifetime within the dot becomes so large it exceeds the inelastic scattering time. In such a situation resonant coherent effects decrease with the result that the Coulomb oscillations do not increase in height close to pinch-off.
We also find that the peak widths do not decrease as $G$ decreases. Generally for $G>2 e^{2} / h$, it is expected that the presence of a fully transmitted $1 \mathrm{D}$ channel might cause mode mixing between 1D channels in the quantum dot which smears out charging effects. However since our samples are fabricated on an ultra high-quality HEMT it is likely that there is little $1 \mathrm{D}$ mode mixing such that the level broadening for Coulomb oscillations is similar for both cases when $G<2 e^{2} / h$ and $2 e^{2} / h<G<4 e^{2} / h$.

Having defined a quantum dot, we now calculate the dot size and the number of electrons it contains following the method described in Ref. 15]. For $V_{S G}=-0.5 \mathrm{~V}$, $V_{F 1}=-1.941 \mathrm{~V}$, and $V_{F 3}=-1.776 \mathrm{~V}$, we observe Aharonov-Bohm type oscillations as a function of applied perpendicular magnetic field [16] with a period $\Delta B$ of $14.7 \mathrm{mT}$, giving a dot area $A$ of $2.81 \times 10^{-13} \mathrm{~m}^{2}$. Using the split-gate to change the dot area at a constant magnetic field of $0.8 \mathrm{~T}$, the Aharonov-Bohm period of oscillations $\Delta V_{S G}^{A B}$ was measured to be $8.772 \mathrm{mV}$ [17. Thus $\Delta V_{S G}^{A B} / \Delta A=1.697 \times 10^{12} \mathrm{Vm}^{-2}$. Each CB oscillation corresponds to removing an electron from the dot so that the reciprocal of the CB period $\Delta N / \Delta V_{S G}^{C B}$ is $263.3 \mathrm{~V}^{-1}$. From the product of these two terms we obtain the local carrier density in the dot to be $4.468 \times 10^{14} \mathrm{~m}^{-2}$. Combining this value with the $\operatorname{dot}$ area $A$ gives the number of electrons in the $\operatorname{dot} N \approx 126$. From the local Fermi energy $E_{F}^{l o c}$ and the number of electrons within the dot, we estimate the $0 \mathrm{D}$ confinement energy $E_{F}^{\text {loc }} / N$ to be at most $12.4 \mu \mathrm{eV}$, comparable to the thermal smearing at $50 \mathrm{mK}$. The reason for this is due to the large dimensions of our sample. Therefore electron transport through our quantum dot can be described in terms of a classical Coulomb charging picture where the $0 \mathrm{D}$ quantum confinement energy is much smaller than the Coulomb charging energy, similar to the case of a metal.

As shown in Fig 2, for $G<2 e^{2} / h$, the conductance oscillations persist up to $T=1 \mathrm{~K}$. The oscillations for $G>2 e^{2} / h$ have a strong temperature dependence and become indistinguishable above $T=410 \mathrm{mK}$. Note that the thermal broadening $k_{B} T$ at this temperature is still much larger than the estimated 0D quantum confinement energy, excluding an interpretation that conductance oscillations for $G>2 e^{2} / h$ are due to tunnelling through $0 \mathrm{D}$ states in the quantum dot. To determine the total capacitance between the dot and the gates of the sample, we measured the conductance oscillations by varying the voltage on the different gates, while keeping the voltages on the remaining gates fixed. From this we obtain $\Delta V_{F 1}=23.806 \mathrm{mV}, \Delta V_{F 2}=8.683 \mathrm{mV}$, $\Delta V_{F 3}=25.894 \mathrm{mV}$, and $\Delta V_{S G}=3.593 \mathrm{mV}$. According to this the total gate-dot capacitance $C_{g}$ is estimated to be $7.579 \times 10^{-17} \mathrm{~F}$. Neglecting the capacitance between the dot and the 2DEG reservoirs, we calculate the Coulomb charging energy $e^{2} / C_{g}$ to be $0.211 \mathrm{meV}$, comparable to the thermal broadening at $T \approx 2 \mathrm{~K}$, which is consistent with the observation that close to pinch-off 
Coulomb oscillations persist up to $1 \mathrm{~K}$.

In order to study the unexpected presence of periodic conductance oscillations for $G>2 e^{2} / h$ in more detail, we have measured their dependence on barrier transparency. Figure 3 (a) shows $G\left(V_{S G}\right)$ as $V_{F 1}$ and $V_{F 3}$ are simultaneously decreased, thus increasing barrier height (decreasing barrier transparency) at zero magnetic field. Figure 3 (b) is a continuation of Fig. 3 (a) at even more negative finger gate voltages. We number peaks in $G\left(V_{S G}\right)$ counted from pinch-off. Note that at pinch-off, we estimate that there are still $\approx 70$ electrons within the dot. Consider the sixth single electron tunnelling peak counted from pinch-off. It is evident that as the barrier heights are raised by making the gate finger voltages more negative, the peak height decreases, and the peak occurs at a more positive $V_{S G}$, i.e., where the channel is wider, as indicated by the dashed line. Thus effectively we are keeping the number of electrons within the dot constant while changing the dot shape. We note that the first ten tunnelling peaks counted from pinchoff in Fig. 3 (a) gradually become indistinguishable as the finger gate voltages are made more negative. This is due to the increasing barrier thickness such that tunneling conductance becomes immeasurably small 15. Over the whole measurement range, we can follow up to 48 conductance tunnelling peaks at various $V_{F 1}\left(V_{F 3}\right)$ and are thus able to study their barrier transparency dependence. Note that the observed conductance oscillations for $G>2 e^{2} / h$ have the same period as that of the oscillating features for $G<2 e^{2} / h$. Most importantly, as shown in Fig. 3 (a) and (b) peaks 31-48, where $G>2 e^{2} / h$ (shown in the uppermost curve), all gradually evolve into conductance oscillations for $G<2 e^{2} / h$ due to Coulomb charging [7 9] as the barrier heights and thickness increase. This result strongly suggests that the conductance oscillations (for peak 31-48 in the uppermost curve shown in Fig. 3 (a) ) and the oscillations shown in the lowermost curves (Fig 3 (b)) are of the same physical originCoulomb charging, compelling experimental evidence for charging effects in the presence of fully transmitted 1D subbands at zero magnetic field.

Finally, we ask the following question: Why is evidence for Coulomb oscillations for $G>2 e^{2} / h$ at zero magnetic field only observed in our system? The reason for this is unclear at present but we speculate that in our case, the overlaying finger gates introduce abrupt tunnel barriers. This is in contrast to work where the tunnel barriers were produced by two quantum point contacts in which smoothly varying potentials are introduced close to the entrant and the exit to the dot. The abrupt tunnel barriers in our case, might reduce the adiabaticity of the transmission of $1 \mathrm{D}$ channels, thereby enhancing electron back-scattering within the dot. This could cause the Coulomb charging effects to be more pronounced in our system as the tunneling $1 \mathrm{D}$ channels for $G>2 e^{2} / h$ are confined within the open dot. Another possible reason is that there is very little inter-1D-subband scattering in our case compared with other systems as endorsed by the lack of resonant features when the tunnel barriers are transparent.

In conclusion, we have presented low-temperature experimental results on an open quantum dot electrostatically defined by a split-gate, and overlaying finger gates which introduce tunnel barriers. Periodic and continuous oscillations superimposed upon ballistic conductance steps are observed even when the conductance through the quantum dot is greater than $2 e^{2} / h$. At zero magnetic field, a direct transition of conductance oscillations for $G>2 e^{2} / h$ to those for $G<2 e^{2} / h$ due to Coulomb charging effects is observed with decreasing barrier transparencies. The temperature dependence of the observed oscillating features for $G>2 e^{2} / h$ excludes the interpretation that they are due to tunneling through single-particle confinement energy states within the dot. Both results suggest that at zero magnetic field charging effects can occur in the presence of a fully transmitted 1D channel, in contrast to the current experimental and theoretical understanding of Coulomb charging.

This work was funded by the UK EPSRC, and in part, by the US Army Research Office. We thank C.H.W. Barnes and V.I. Talyanskii for discussions. G.H.K. acknowledges financial assistance from Clare College.

\section{References}

[1] T.J. Thornton et al., Phys. Rev. Lett. 56, 1198 (1986).

[2] C.G. Smith et al., J. Phys. C 21, L893 (1988).

[3] U. Meirav, M.A. Kastner, and S.J. Wind, Phys. Rev. Lett. 65, 771 (1990).

[4] P.L. McEuen et al., Phys. Rev. Lett. 66, 1926 (1991).

[5] J. Weis et al., Phys. Rev. Lett. 71, 4019 (1994).

[6] R.C. Ashoori et al., Phys. Rev. Lett. 68, 3088 (1992).

[7] A.A.M. Staring et al., Physica B 175, 226 (1991).

[8] L.P. Kouwenhoven et al., Z. Phys. B 85, 367 (1991).

[9] J.G. Williamson et al., in Nanostructures and Mesoscopic Systems, edited by W.P. Kirk and M.A. Reed, (Academic Press, 1992) p. 255.

[10] L.W. Molenkamp, K. Flensberg and M. Kemerink, Phys. Rev. Lett. 75, 4282 (1995).

[11] C.-T. Liang et al., Phys. Rev. B 55, 6723 (1997).

[12] I.V. Zozoulenko and K.-F. Berggren, Phys. Rev. B 54, 5823 (1996).

[13] I. Zailer et al., Semicond. Sci. Technol. 11, 1235 (1996).

[14] M. Buttiker, Phys. Rev. B 41, 7906 (1990).

[15] M. Field et al., Phys. Rev. Lett. 70, 1311 (1993).

[16] B.J. van Wees et al., Phys. Rev. Lett. 62, 2523 (1989).

[17] R.J. Brown et al., J. Phys. Cond. Matter 1, 6291 (1989).

\section{Figure Captions}


FIG. 1. Trace 1: $G\left(V_{S G}\right)$ for all finger gates at $0 \mathrm{~V}$. Trace 2 to 4: $G\left(V_{F 1}\right)$ (in solid line), $G\left(V_{F 3}\right)$ (in dashed line) and $G\left(V_{F 2}\right)$ (in dotted line) for $V_{S G}=-1.132 \mathrm{~V}$. The inset shows an scanning electron micrograph of a typical device. The brightest regions correspond to finger gates with joining pads, labelled as F1, F2, and F3 lying above the split-gate (labelled as SG), with an insulating layer of crosslinked PMMA in between.

FIG. 2. $G\left(V_{S G}\right)$ for $V_{F 1}=-1.941 \mathrm{~V}, V_{F 2}=0 \mathrm{~V}$, and $V_{F 3}=-1.776 \mathrm{~V}$ at various temperatures $T$. From left to right: $T=1,0.5,0.45,0.41,0.35,0.3,0.26,0.2$, $0.18,0.17,0.15,0.11,0.09,0.065$ and $0.05 \mathrm{~K}$. Curves are successively displaced by a horizontal offset of $0.02 \mathrm{~V}$ for clarity.
FIG. 3. (a) $G\left(V_{S G}\right)$ at various voltages applied on F1 and F3 at zero magnetic field. From top to bottom: $V_{F 1}=-1.907 \mathrm{~V}$ to $-1.965 \mathrm{~V}$ in $2 \mathrm{mV}$ steps $\left(V_{F 3}=-1.733 \mathrm{~V}\right.$ to $-1.805 \mathrm{~V}$ in $2.5 \mathrm{mV}$ steps $)(\mathrm{b})$ Continuation of figure 3 (a). From top to bottom: $V_{F 1}=$ $-1.965 \mathrm{~V}$ to $-2.023 \mathrm{~V}$ in $2 \mathrm{mV}$ steps $\left(V_{F 3}=-1.805 \mathrm{~V}\right.$ to $-1.8775 \mathrm{~V}$ in $2.5 \mathrm{mV}$ steps) Curves are successively offset by $(0.0344)\left(2 e^{2} / h\right)$ for clarity. Conductance tunneling peaks are numbered to serve a guide to the eye for the evolution of oscillating structures in $G\left(V_{S G}\right)$. The dashed line in Fig. 3 (a) serves as a guide to the eye for the evolution of peak 6 . The measurement temperatures were $50 \mathrm{mK}$. 
Figure 1 C-T Liang et al.

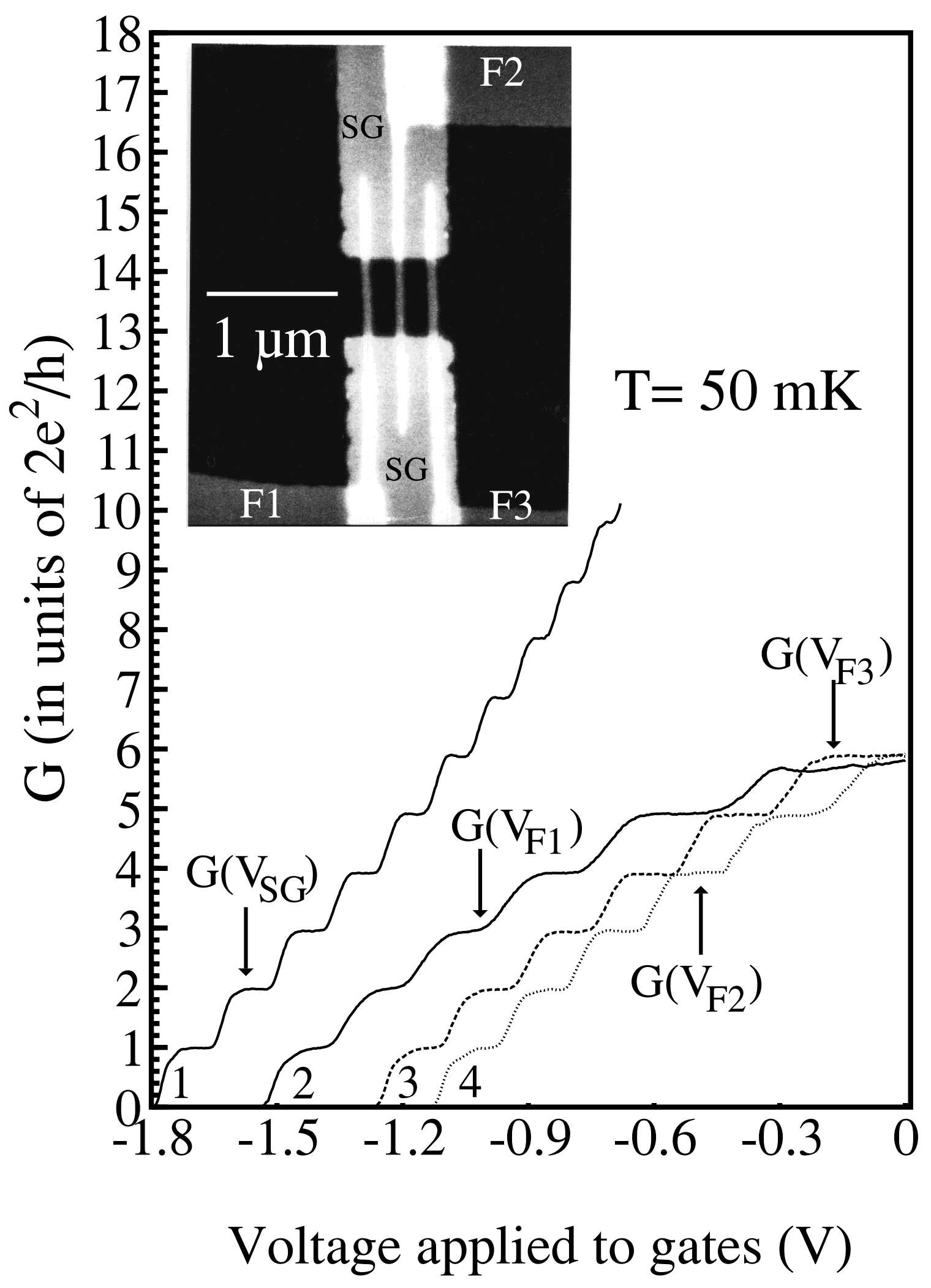


Figure $2 \mathrm{C}$-T Liang et al.

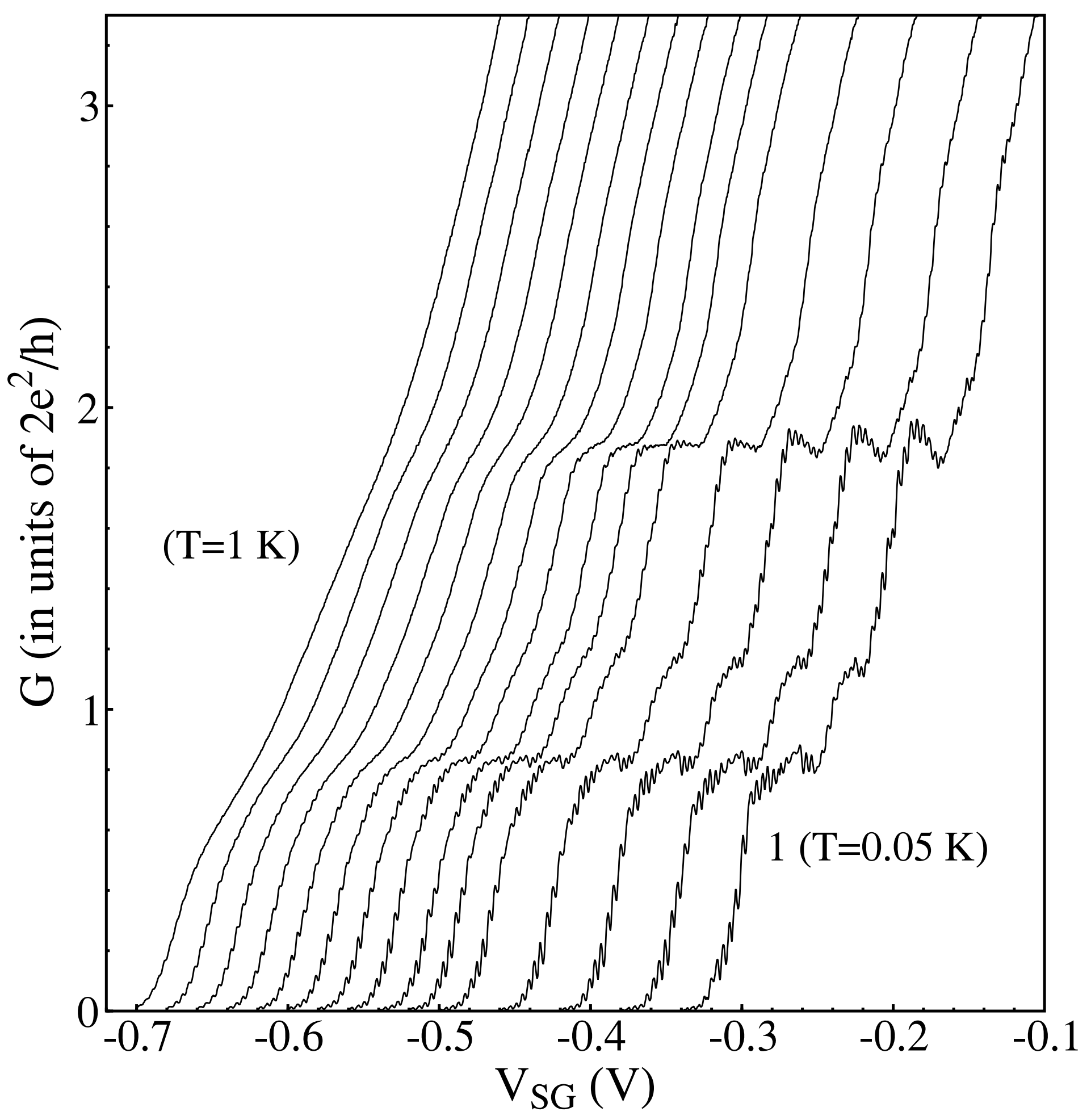


Figure 3 (a) C-T Liang et al.

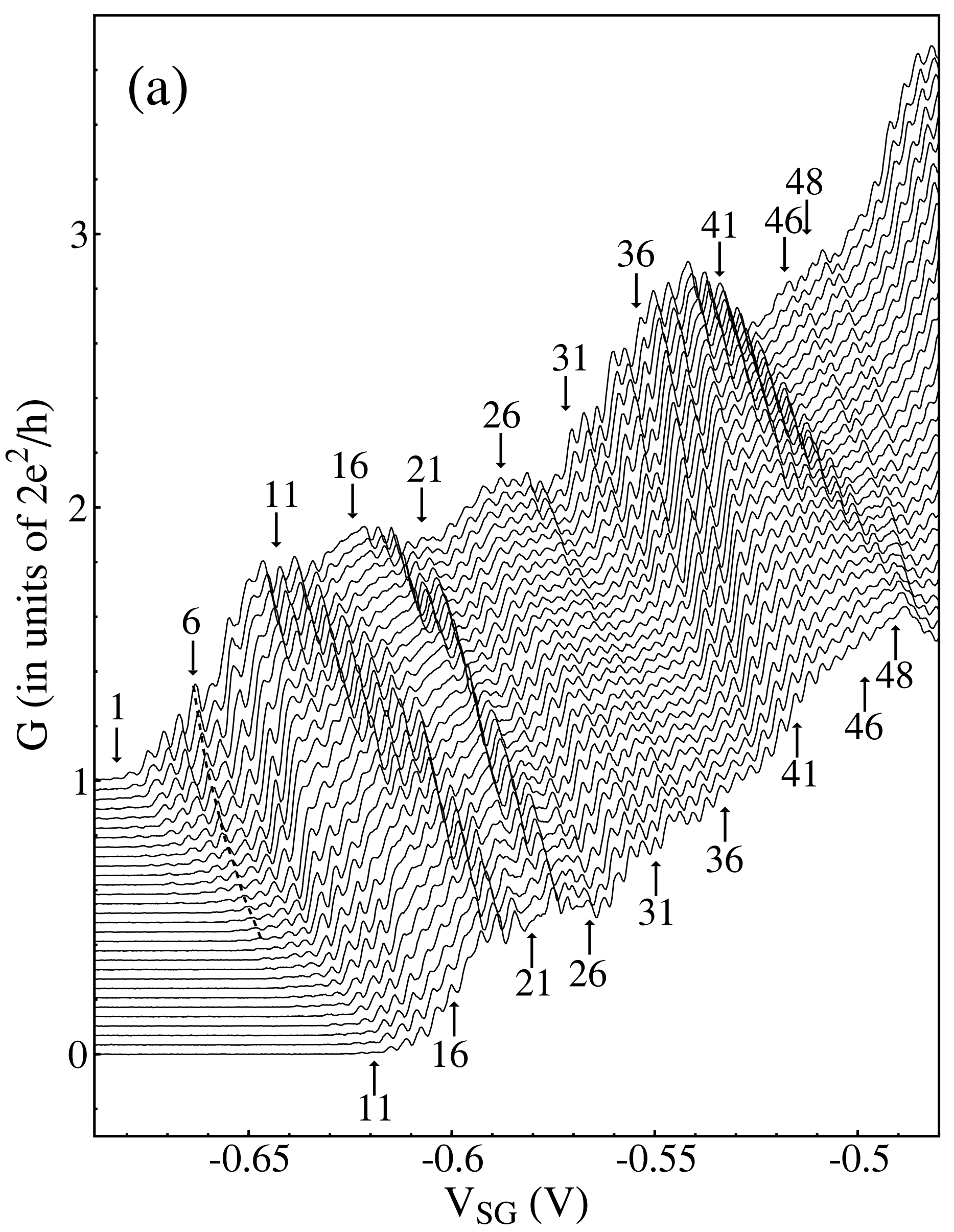


Figure 3 (b) C-T Liang et al.

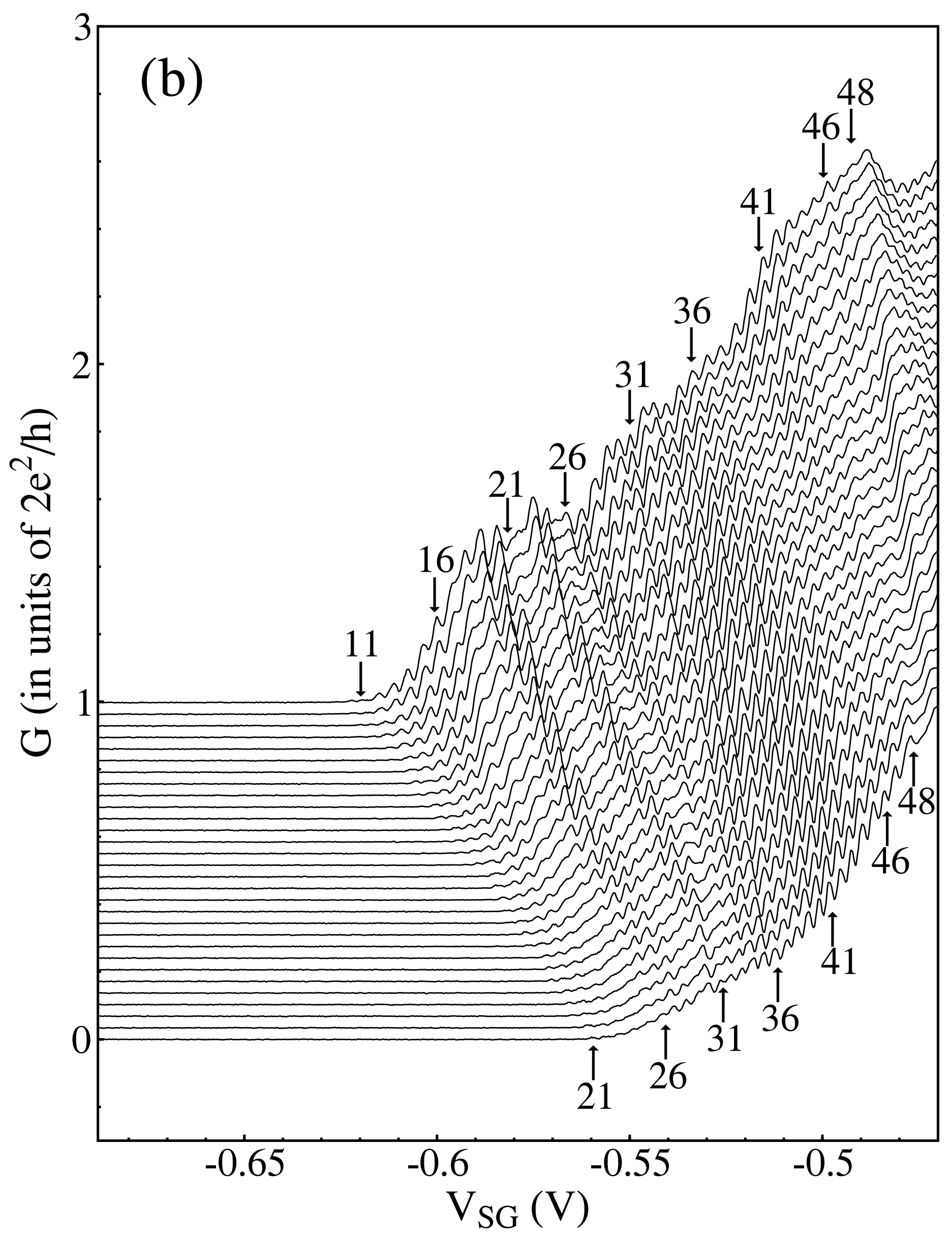

